\title{
Nonlinear, multiple-input modeling of cerebral autoregulation using Volterra Kernel estimation
}

\author{
H. Kouchakpour, R. Allen, D.M. Simpson
}

\begin{abstract}
Autoregulation refers to the automatic adjustment of blood flow to supply the required oxygen and glucose and remove waste, in proportion to the tissue's requirement at any instant of time. For the brain, cerebral autoregulation is an active process by which cerebral blood flow is controlled at an approximately steady level despite changes in the arterial blood pressure. Robust assessment of the cerebral autoregulation by a model that characterizes this system has been the goal of many studies, searching for techniques that can be used in clinical scenarios to detect potentially dangerous impairment of control. Multiple input, single output (MISO) models can be used to assess autoregulation, and system parameters can be estimated from spontaneous beat-to-beat variations in arterial blood pressure (ABP) and breath-by-breath end-tidal carbon dioxide $\left(\mathrm{P}_{\mathrm{ETCO} 2}\right)$ as inputs, and cerebral blood flow velocity (CBFV) as the output .In this study a non-linear, multivariate approach, based on Volterra-type kernel estimation models is employed. The results are compared with linear models as well as nonlinear single-input single-output (SISO) models. The normalized mean squared error was used as the criteria of performance of each model in assessing cerebral autoregulation. Our simulation results indicate that for relatively short signals (around $300 \mathrm{sec}$ ), nonlinear, multipleinput models based on Volterra systems performed best, though the benefit varied considerably between subjects. When using a fixed model for all recordings, a linear SISO model with ABP as input provided the smallest average modeling error.

Keywords- Cerebral Autoregulation, Non-linear analysis, physiological systems, Blood pressure, CO2, Blood flow, Volterra Kernel Models, Laguerre- Volterra networks (LVNs).
\end{abstract}

\section{INTRODUCTION}

C erebral autoregulation (CA) refers to the ability of the brain to control the diameter of small blood vessels to maintain cerebral perfusion relatively constant, despite changes in blood pressure (BP), in order to protect the brain from injury due to insufficient or excessive blood flow resulting from a sudden drop or surge in arterial blood pressure (ABP) [1]

Over the past two decades most of the studies carried out on cerebral autoregulation have used non-invasive methods to measure cerebral blood flow velocity (CBFV- employing Transcranial Doppler ultrasound) in response to transient changes in ABP [2]. This is known as dynamic cerebral autoregulation (dCA), in contrast to static cerebral

H. Kouchakpour. Author is with the ISVR, Southampton University, SO17 1BJ, UK (phone: 44-7792-591411; e-mail: hk803@soton.ac.uk).

R. Allen. Author is with the ISVR, Southampton University, SO17 1BJ, UK (email: ra@isvr.soton.ac.uk)

D.M. Simpson. Author is with the ISVR, Southampton University, SO17 1BJ, UK (email: ds@isvr.soton.ac.uk) autoregulation, where steady-state responses following changes in baseline level of pressure are measured.

In order to assess dCA a few methods to induce large, rapid changes in $\mathrm{ABP}$ have been proposed in the literature: thigh cuff release produces a sudden step decrease in BP [2], lower body negative pressure can give sinusoidal variation in $\mathrm{CBFV}$ [3], the valsalva maneuver provokes characteristic change in BP and CBFV [4], and periodic breathing, squatting and head-up tilt [5], [6], [7] have all been used. Spontaneous variations in blood pressure and pCO2 (an example is shown in fig 1) may also provide sufficient excitation to assess autoregulation, while minimizing interference with the patient which is clearly desirable for clinical studies.

After inducing changes in $\mathrm{BP}$ and $\mathrm{CBFV}$, the relationship between these variables has to be quantified. Dynamic cerebral autoregulation is a frequency dependent phenomenon and non-linear behavior has been noted [8]. However most of the work done in this area is based on the assumption of linearity, and hence the frequency and impulse responses have been used to characterize the dynamic relationship between $\mathrm{ABP}$ and CBFV [1]. The phase shift and gain between spontaneous variation of $A B P$ and CBFV from transfer function analysis (TFA) have shown the high-pass filter characteristics of cerebral autoregulation [1], [9]. In the time domain, IIR (autoregressive (AR), autoregressive moving average (ARMA)) and FIR linear filters have been used to model the system. Methods such as the ARi (autoregulatory index) [4] have been proposed to assess autoregulation, using a set of 10 pre-defined linear filters, grading the responses from excellent ('9') to absent (' 0 '). Although linear models can provide relatively good results, evidence suggests the existence of nonlinearity in the autoregulatory system [8].

Apart from nonlinearity, there are other physiological variables, including $\mathrm{pCO}_{2}$, brain metabolic activities, haematocrit and sympathetic tone that affect the blood flow and its regulation [1], [10]. $\mathrm{pCO}_{2}$ can be measured noninvasively by breath-to-breath measurements of end-tidal $\mathrm{CO}_{2}$ concentrations. Hypercapnia causes vasodilatation, while hypocapnia provokes vasoconstriction. In addition, hypercapnia causes impairment of autoregulation. In recent studies it has been shown that the spontaneous variation of $\mathrm{CO}_{2}$ has a significant effect in the very low frequency band $(<0.04 \mathrm{~Hz})$ of $\mathrm{CBFV}$, as determined by applying nonlinear methods [11], [12]. However, the benefit of non-linear modeling, and which model might be most appropriate when data is relatively short, as is commonly the case in research 
and clinical studies, has not been firmly established.

In order to address these issues, in this work the dynamic relationship between $\mathrm{CBFV}, \mathrm{MABP}$, and $\mathrm{pCO}_{2}$ is investigated through non-linear models, using Wiener Laguerre estimation methods. The results obtained from these multivariate, nonlinear models are compared to single input (just MABP) linear and nonlinear models, and multivariate linear models. Recommendations are provided for selecting optimal model orders.
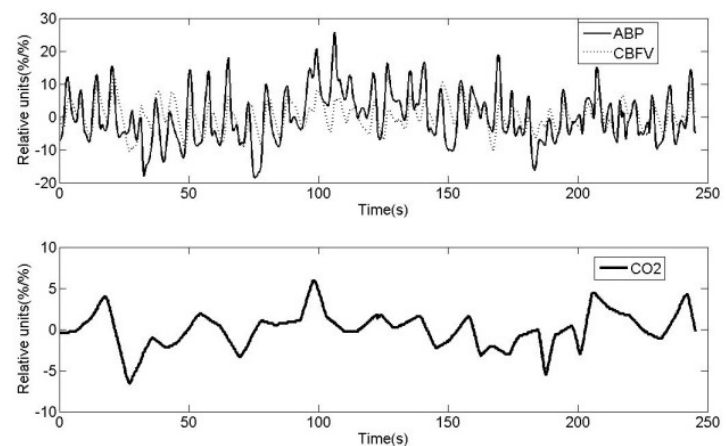

Fig.1. Representative segments of $\mathrm{ABP}, \mathrm{CBFV}$ and $\mathrm{CO}_{2}$, for one measurement. Top: Cerebral Blood Flow Velocity (CBFV) and Arterial Blood Pressure (ABP), Bottom: $\mathrm{CO}_{2}$. The phase lead characteristics of cerebral autoregulation can be seen in the top figure.

\section{DATA COLLECTION AND PRE-PROCESSING}

The data used in this study was kindly provided by Profs. D.H. Evans and R. Panerai, and Dr. S.T. Deverson and was collected at the Leicester Royal Infirmary (Leicester, UK). Fifteen healthy volunteers (age $32 \pm 8.8$ years) were involved in this study and the study was approved by the Leicestershire Research Ethics Committee. All the measurements were collected with the volunteers in the supine position with their head elevated. Transcranial Doppler Ultrasound (Scimed QVL-120,) was used to measure middle cerebral artery velocity using a $2 \mathrm{MHz}$ transducer, held in position by an elastic headband. Simultaneously arterial blood pressure (ABP) was noninvasively measured using a finger cuff device (Ohmeda 2300 Finapress monitor).

The signals were pre-processed off-line. The maximum velocity envelope from the spectra of the Doppler signal was extracted using a microcomputer-based analyzer that performs a fast Fourier transform (FFT) every $5 \mathrm{~ms}$. The ABP signals were digitized at $200 \mathrm{~Hz}$. Short periods of evident artefact as well as any spikes on the signals were removed by linear interpolation and the signals (ABP, CBF) were low pass filtered with an eighth-order Butterworth digital filter (applied forward and reverse to give zero phase shift) with a cut-off frequency of $20 \mathrm{~Hz}$. The start of each heart cycle was automatically identified (with visual correction) from the ABP signal, after which the average $\mathrm{ABP}$ and CBFVs from the right and left MCA were calculated for each heartbeat. This time series was then interpolated with a third-order polynomial, and sampled at a constant rate of $5 \mathrm{~Hz}$.

\section{A. Data Analysis}

For each measurement, data segments of approximately
$300 \mathrm{~s}$ in duration were available. The recordings were converted to a percent change with respect to the mean value of each data segment, in order to remove the dependence on inter-individual variations in mean level. The pre-processed (\% change) $\mathrm{ABP}, \mathrm{CBFV}$ and $\mathrm{pCO}_{2}$ are referred to as $\mathrm{P}(\mathrm{t})$, $\mathrm{V}(\mathrm{t})$ and $\mathrm{CO}_{2}(\mathrm{t})$, respectively, from this point on.

\section{B. Mathematical modeling}

The Volterra-Wiener modeling has been widely used in nonlinear modeling of physiological systems. In this work, a multi-input, general Volterra-Laguerre model of cerebral autoregulation is used to get an understanding of the effects of both $\mathrm{MABP}$ and $\mathrm{pCO}_{2}$ changes on $\mathrm{CBFV}$ variations:

$$
\begin{aligned}
& V(t)=k_{0,0}+\sum_{j_{1=1}}^{L_{1}} c_{1,0}\left(j_{1}\right) v_{j_{1}}{ }^{(1)}(\mathrm{t})+\sum_{j_{1=1}}^{L_{2}} c_{0,1}\left(j_{1}\right) v_{j_{1}}{ }^{(2)}(\mathrm{t}) \\
& +\sum_{j_{1=1}}^{L_{3}} \sum_{j_{2}=1}^{L_{3}=1} c_{2,0}\left(j_{1}, j_{2}\right) v_{j_{1}}{ }^{(1)}(\mathrm{t}) v_{j_{2}}{ }^{(1)}(\mathrm{t}) \\
& +\sum_{j_{1=1}}^{L_{4}} \sum_{j_{2}=1}^{L_{4}} c_{0,2}\left(j_{1}, j_{2}\right) v_{j_{1}}{ }^{(2)}(\mathrm{t}) v_{j_{2}}{ }^{(2)}(\mathrm{t}) \\
& +\sum_{j_{1=1}}^{L_{5}} \sum_{j_{2}=1}^{L_{5}} c_{1,1}\left(j_{1}, j_{2}\right) v_{j_{1}}{ }^{(1)}(\mathrm{t}) v_{j_{2}}{ }^{\left({ }^{2}\right)}(\mathrm{t})+\ldots
\end{aligned}
$$

$k_{m, n}\left(\tau_{1}, \ldots, \tau_{m+n}\right)=$ $\sum_{j_{1=1}}^{L_{l}} \ldots \sum_{m+n=1}^{L_{l}} c_{m, n}\left(j_{1}, \ldots, j_{m+n}\right) b_{j_{1}}\left(\tau_{1}\right) \ldots b_{j_{m+n}}\left(\tau_{m+n}\right)$

Where (1) and (2) refer to the inputs $\mathrm{P}(\mathrm{t})$ and $\mathrm{CO}_{2}(\mathrm{t})$ respectively at time $t$. The unknown parameters $k_{n}\left(\tau_{1}, \tau_{2}, \ldots, \tau_{m+n}\right), \quad c_{m, n}\left(j_{1}, \ldots, j_{m+n}\right), b_{j_{m+n}}\left(\tau_{m+n}\right)$ in above equations are the Volterra kernels (to be estimated from input-output data), the expansion coefficients of $k_{m, n}$ and the $j t h$ basis function respectively. The first order Volterra kernels $k_{1,0}, k_{0,1}$ are the linear components of the system dynamics, whilst the higher order kernels $\left(k_{1,1}, k_{2,0}, k_{0,2}, \ldots\right)$ are the nonlinear components of the system. In most physiological systems the second or third order Volterra models are considered sufficient to describe the system [13]. In this work only kernels of up to second order $\left(k_{0,0}, k_{1,0}, k_{0,1}, k_{2,0}, k_{0,2}, k_{1,1}\right)$ are used due to the size of available data segments. With higher orders, the number of parameters increases rapidly and quickly exceeds the number of samples in any reasonable length recording. It has to be noted that the $k_{2,0}, k_{0,2}$ are called the self-kernels and $k_{1,1}$ is known as the cross-kernels.

There are different methods for estimating the discretized Volterra kernels and amongst them the Volterra-equivalent network in the form of the Laguerre-Volterra Network (LVN) has shown to be the most efficient [14] of the kernel expansion approaches and provides the best model of nonlinear systems with short segments of data available [10].The Laguerre-Volterra network (LVN) is a combination of artificial neural networks with the Laguerre expansion technique (LET) [8]. The LVN for bivariate models consists of one input layer with two separate Laguerre filter banks (may be the same set of filters) and a hidden layer with $\mathrm{H}$ hidden units using polynomial activation functions (see fig 2). The LVN model consists of individual dual-input static nonlinearities associated with each input-output pair. 


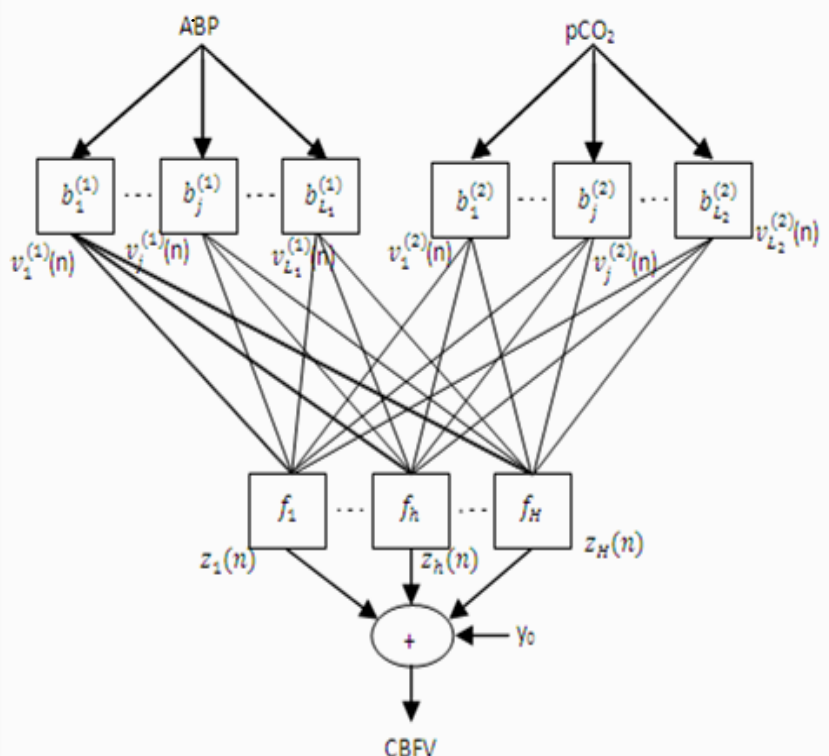

Fig.2. The Volterra Equivalent network with two-inputs, with each input pre-processed through a different filter $\operatorname{bank}\left(b_{j}^{(i)}\right)$ and respectitive filter bank outputs are fed into the hidden units of te hidden layer with polynomial activation functions $\left(f_{h}\right)$, and the output is calculated as the summation of the outputs of the hidden units $\left(z_{h}(\mathrm{n})\right)$ and and offset $y_{0}[13]$.

The output of the $\mathrm{j}^{\text {th }}$ filterbank is given by the convolution:

$v_{j}^{(1)}(\mathrm{n})=\sum_{m=0}^{M-1} b_{j}^{(i)}(m) x_{i}(n-m)$

$y(n)=y_{0}+\sum_{1}^{H} z_{h}(n)$

$z_{h}(n)=f_{h}\left[u_{h}(n)\right]=\sum_{q=1}^{Q} c_{h, q} u_{h}^{q}(n)$

Where $\mathrm{M}$ is the memory (length of the impulse response) and $b_{j}^{(i)}$ (Discrete Laguerre function (DLF)) denotes the basis function that is the impulse response of the jth filter in the ith filterbank, $Q$ is the order of nonlinearity and $f_{h}$ is the polynomial static nonlinearity [13]. An important parameter in calculating DLF is the positive $\alpha$ value which determines the rate of exponential decline of these functions. This value was chosen for each measurement based on the number of filterbanks for each kernel (if they exist) and the length of the impulse response.

For each set of data and each model, the predicted output (CBFV) was compared to the measured output and the performance was evaluated using the normalized mean square error (NMSE; the difference between the predicted and measured velocity normalized by the mean square of the measured velocity). Evaluation was carried out using crossvalidation, in which model parameters were estimated on one data segment (training set), and NMSE then calculated on a second segment (validation set) from the same recording; both segments were $150 \mathrm{~s}$ long. The procedure was then repeated, swapping training and validation segments. The memory $M$ of the filters for each set of measurements was evaluated using NMSE between the response of CBFV to changes in $\mathrm{ABP}$ as a linear system (just $k_{0,0}, k_{1,0}$, in eq. 2.) and this value was then used in the multivariate and nonlinear models. For LVN to estimate the kernels precisely the number of filterbanks should be large enough. In other words, a reduced of filterbanks would results in LVN being a small sub-set of the solution. In this work we test all the possible combinations of filterbanks for each kernels ( 1 to 15 for linear kernels and 0 to 3 for nonlinear kernels) to ensure the validation of the results based on the criteria of NMSE.

\section{RESULTS}

Based on the sequence of $\mathrm{CO} 2$ levels (normo, hyper and normo-capnia), three recordings from fifteen volunteers were analyzed, and the model that generated the best prediction in the validation set for each measurement was identified. The impulse response length for each recording was calculated individually from the single-input linear model (ABP-CBFV) and then, this impulse response was used to estimate the filterbank orders for each of the models. In each model the filterbanks for each kernels varied from ' 0 ' (absence of that kernel) to the maximum number of filterbanks for that kernel (thirty for linear and 3 for nonlinear kernels). It was found that the maximum number of filterbanks for the nonlinear kernels was two and for linear kernels this was twenty.

The average output prediction achieved in the training and validation sets for linear, nonlinear single-input (ABP), and linear, nonlinear two-input $\left(\mathrm{ABP}, \mathrm{pCO}_{2}\right) \mathrm{LVN}$ models are presented in Table 1. For all measurements better performance was observed for training data, as expected from theory [13]. The results show that by adding $\mathrm{pCO}_{2}$ the NMSE of the LVN model prediction in the validation data reduces compared to single-input linear and nonlinear models. The average reduction in NMSE\% from the singleinput, linear model and single-input, nonlinear model to twoinput nonlinear models are $10.38 \%$ and $9.0 \%$ in validation respectively, indicating the multivariate and nonlinear natures of cerebral regulation. However, the results suggested that for 8 measurements in the first half training, and 3 measurements in the second half training, linear single-input (ABP) gave the best performance in terms of the NMSE.

The first order (linear), second-order (nonlinear) kernels and cross-kernels for one subject are shown in fig 3 . The results shows that the effect of $\mathrm{CO}_{2}$ is slower compared to $\mathrm{ABP}$, as expected [10], probably due to transport phenomena.

The second-order self and cross-kernels showed that nonlinearity exists in the system and from literature [10] we know it affects mostly the low frequency band (below 0.1 Hz. Further analysis indicates that the cross-kernels (interaction between $\mathrm{ABP}$ and $\mathrm{CO}_{2}$ ) had a stronger effect on the NMSE than either of the second order self-kernels.

In practice it is probably desirable to choose a fixed order for all recordings. In the current study, the lowest average NMSE across all recordings was obtained for the $4^{\text {th }}$ order SISO linear model with impulse response length of 5.4 seconds, with only ABP as input. 

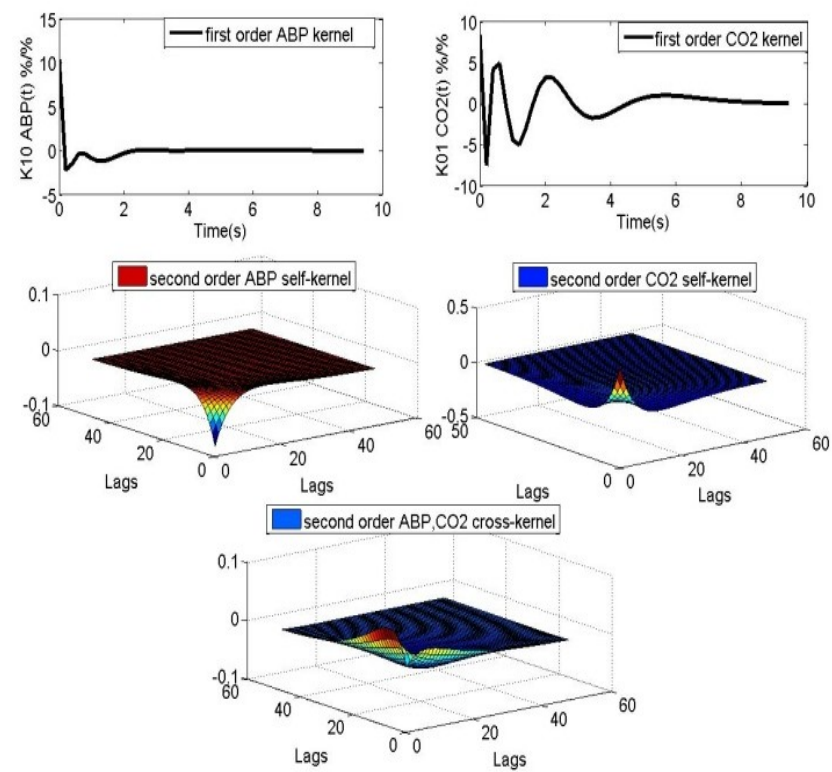

Fig.3. Top row: First order Kernel estimated for 1 measurement set for ABP and $\mathrm{CO} 2\left(k_{10}, k_{01}\right)$, Second row, left: Second-order self-kernel ABP estimated $\left(k_{20}\right)$, right: Second-order self-kernel $\mathrm{CO}_{2}$ estimated $\left(k_{02}\right)$, Bottom row: Second-order cross-kernel $\mathrm{ABP}-\mathrm{CO}_{2}$ estimated $\left(k_{11}\right)$ with filterbanks of 7, 7,2,2,2 respectively. It can be seen that $\mathrm{CO}_{2}$ has much slower response compared to ABP (top row impulse responses).

TABLE 1 Different Model NMSE\% comparison

\begin{tabular}{|c|c|c|c|c|}
\hline Model & $\begin{array}{l}\text { Training } \\
\text { NMSE\% }\end{array}$ & $\begin{array}{l}\text { Validation } \\
\text { NMSE\% }\end{array}$ & $\mathrm{p}$-value & $\begin{array}{c}\text { Average } \\
\text { number } \\
\text { of } \\
\text { paramete } \\
\text { rs used }\end{array}$ \\
\hline $\begin{array}{l}k_{10} \quad \text { (linear, } \\
\text { single-input) }\end{array}$ & $\begin{array}{r}16.2105 \\
\pm 8.5186\end{array}$ & $\begin{array}{c}16.2825 \pm 7 \\
0809\end{array}$ & 0.0035 & 6 \\
\hline $\begin{array}{l}k_{10}, k_{01} \text { (linear } \\
\text { two-input) }\end{array}$ & $\begin{array}{r}14.4922 \\
\pm 7.3073\end{array}$ & $\begin{array}{l}15.2837 \pm 6 \\
4416\end{array}$ & $\begin{array}{c}0.0009 \\
6\end{array}$ & 7 \\
\hline $\begin{array}{l}k_{10}, k_{20} \text { (nonline } \\
\text { ar; self-kernels, } \\
\text { single-input) }\end{array}$ & $\begin{array}{l}15.1327 \\
\pm 7.4408\end{array}$ & $\begin{array}{c}16.1878 \pm 7 \\
1721\end{array}$ & 0.0024 & 6 \\
\hline $\begin{array}{l}k_{10}, k_{11} \text { (nonline } \\
\text { ar; cross- } \\
\text { kernels, two- } \\
\text { inputs) }\end{array}$ & $\begin{array}{l}15.1780 \\
\pm 7.4861\end{array}$ & $\begin{array}{c}16.1458 \pm 6 . \\
9756\end{array}$ & 0.0049 & 6 \\
\hline $\begin{array}{l}k_{10}, \\
k_{01}, k_{20}, k_{02}, k_{11} \\
\text { (nonlinear; } \\
\text { self-kernels, } \\
\text { cross-kernels, } \\
\text { two-inputs) }\end{array}$ & $\begin{array}{r}14.1697 \\
\pm 7.7042\end{array}$ & $\begin{array}{c}14.5993 \pm 6 . \\
1290\end{array}$ & 0.0032 & 9 \\
\hline
\end{tabular}

\section{CONCLUSION}

In this work the contribution of $\mathrm{pCO}_{2}$ and $\mathrm{ABP}$ to spontaneous changes to CBFV fluctuation with LVN models were studied, by estimating the minimum error with different model orders. It was found that, by adding $\mathrm{CO}_{2}$ as a secondary input to $\mathrm{ABP}$, somewhat superior predictive performance and more accurate models of $\mathrm{CBF}$ regulation can be achieved. This work illustrates the importance of the multivariate characteristics of $\mathrm{CBF}$ regulation and of the cross-kernels between the inputs ( $\mathrm{ABP}$ and $\mathrm{CO}_{2}$ ). However, the benefit of non-linear modeling was not evident in all cases, which may be due to inter-individual differences in brain blood flow control. Furthermore, the best average performance was obtained by a $4^{\text {th }}$ order (number of filterbanks) linear SISO model with impulse response length of 5.4 seconds, with only ABP as input.

\section{ACKNOWLEDGMENT}

We would like to thank Prof. R. Panerai, Prof. D. Evans and Dr. Stephanie Foster (Leicester Royal Infirmary/University of Leicester) for providing the anonymized data used in this study, collected using equipment developed by Dr. L. Fan (Leicester Royal Infirmary), and also EPSRC for funding this project.

\section{REFERENCES}

[1] R. Panerai, "Assessment of cerebral pressure autoregulation in humans -a review of measurement methods" Physiological Measurements, 1998, vol: 305, pp. 15-64.

[2] R. Aaslid, K.F. Lindegaard, w. Sorteberg, H. Nomes, "Cerebral auoregulation dynamics in humans" Stroke, 1989, vol:20, pp:45-52.

[3] B.D. Levine, C.A. Giller, L.D. Lane, J.C. Buckey, C.G. Blomqvist, "Cerebral versus systemic hemodynamics during graded orthostatic stress in humans "Circulation, 1994, vol:90,pp:198-206.

[4] F.P. Tiecks, A.M. Lam, R. Aaslid, D.W. Newell, "Effects of the valsalva manoeuvre on cerebral circulation in healthy adults, A transcranial Doppler study" Stroke, 1995, vol: 26, pp:1386-92.

[5] R.R. Diehl, D. Linden, D. Lucke, P. Berlit, "Phase relationship between cerebral blood flow velocity and blood pressure, A clinical test of autoregulation" Stroke, 1995, vol:26, pp:1801-4.

[6] A.A. Birch, M.J. Dirnhuber, R. Hartley-Davis, F. Iannotti, G. NeilDwyer, "Assessment of autoregulation using sinusoidal lower body negative pressure" Physiological Measurements, 1995, vols: 23, pp:73-83.

[7] J.P. Oblak, M. Zaletel, B. Zvan, T. Kiauta, T. Pogacnik, "the effect of age on cerebrovascular reactivity to cold pressure test and head-up tilt" Acta Neurologica Scandinavica, 2002, vol:106, pp:30-36.

[8] G.D. Mitsis, R. Zhang, B.D. Levine, V.Z. Marmarelis, "Modeling of nonlinear physiological systems with fast and slow dynamics. II. Application to cerebral autoregulation" Annual Biomedical Engineering, 2002, vol: 30, pp: 555-65.

[9] R. Zhang, J.H. Zuckerman, C.A. Giller, B.D. Levine, "Transfer function analysis of dynamic cerebral autoregulation in humans" American Journal of Physiology, 1998, vol:274, pp:233-241.

[10] G.D. Mitsis, V.Z. Marmarelis, M.J. Poulin, P.A. Robbins, "Nonlinear multivariate analysis of dynamic cerebral blood flow regulation in humans" Second Joint EMBS-BMES conference, 2002, vol: 2, pp: $1341-2$

[11] G.D. Mitsis, V.Z. Marmarelis, M.J. Poulin, P.A. Robbins, "Nonlinear modeling of the dynamic effects of arterial pressure and $\mathrm{CO} 2$ variations on cerebral blood flow in healthy humans" IEEE Transactions on Biomedical Engineering, 2004, vol: 51, pp:19321944.

[12] G.D. Mitsis, R. Zhang, B.D. Levine, V.Z. Marmarelis, "Cerebral hemodynamics during orthostatic stress assessed by nonlinear modeling" Physiological Applications, 2005, vol:101, pp:354-366.

[13] D.T. Westwick, R.E. Kearney, "Identification of nonlinear physiological systems" IEEE press/Wiley, Piscataway, NJ, 2003/

[14] V.Z. Marmarelis, "Nonlinear dynamic modelling of physiological systems" IEEE Engineering in Medicine and Biology Society/Wiley, New York, 2004. 